\title{
ESTUDO DA TEORIA DAS FILAS APLICADO A UM SUPERMERCADO NO CARIRI PARAIBANO
}

\author{
Bárbara Valéria Pereira Lins (UFCG) bvpl_08@ hotmail.com \\ D’ávilla Jhonny Lima Santos (UFCG)d.jhonny95@gmail.com \\ Laís Vaz Mendes (UFCG) laisvvaz@gmail.com \\ Mailson Araújo Feitoza Filho (UFCG) feitozafilho22@ hotmail.com \\ Priscilla Kelly Santos de Oliveira (UFCG) priscilla.santos14@hotmail.com
}

\section{Resumo}

O objetivo deste trabalho foi realizar uma modelagem de dados de entrada e análise dos sistemas de filas realizados em um supermercado na região do Cariri Paraibano localizada na cidade de Sumé. A pesquisa contou com o levantamento de dados desenvolvidos de um modelo de otimização de teoria das filas. Como resultado, foi possível observar que o sistema em estudo está superdimensionado, a simulação realizada com três atendentes é considerada desvantajosa por trazerem custos operacionais para organização, foi obtidas propostas de melhorias para dois caixas trará uma maior utilização sem exceder os limites nos dias de altas demandas gerando menor custo para empresa e mantendo um limite de segurança em eventos de altas inesperadas, podendo ainda, aproveitar o funcionário que estaria ocioso em outras funções nesse período.

Palavras-Chaves: Modelagem de dados, Teoria das Filas, Supermercado.

\section{Introdução}

As filas são sistemas comuns a grande maioria das organizações, como farmácias, padarias, supermercados, bem como nos sistemas de manufatura, transporte e sistemas computacionais. Devido à presença rotineira dessas filas, se faz necessária a realização de estudos que visam uma melhor qualidade do serviço prestado independentemente dos seus custos ou uma qualidade moderada (aceitável) com custos reduzidos.

Nesse contexto, a Teoria das Filas busca tratar de problemas de congestionamento de sistemas, bem como os atrasos sofridos pelos usuários do sistema, podendo ser aplicado nas variadas áreas da administração, desde política do atendimento ao cliente, a programação de linhas de montagem (CAIXETA FILHO, 2004).

No que se diz respeito às filas que se formam em caixas rápidos de supermercados, os congestionamentos merecem atenção, visto que os consumidores correlacionam o tempo total 
gasto no supermercado com a falta de organização geral do mesmo (MORABITO; LIMA, 2000).

O trabalho foi elaborado com a justificativa de se desenvolver os conceitos de Teoria das Filas perante a Engenharia de Produção. O objetivo é avaliar os parâmetros de um sistema de fila que se forma no acesso de três canais de atendimento (caixa) de um Supermercado localizado na região do Cariri no estado da Paraíba, verificando se o cenário atual de dimensionamento de instalações, equipamentos e infraestrutura utilizado pela empresa condiz com os propostos após os resultados do trabalho.

\section{Referencial Teórico}

\subsection{Filas}

Para Lovelock e Wright (2002), uma fila é a representação de uma linha de pessoas, veículos, objetos físicos ou intangíveis que aguardam sua vez de serem atendidos. O processo de formação de filas ocorre quando o número de chegadas excede à capacidade do sistema de atendimento e geralmente está relacionado a problemas relativos à administração da capacidade.

\subsection{Teoria das Filas}

Fogliatti e Mattos (2007) definem que um sistema com fila consiste na chegada de usuários que necessitam de um determinado serviço, esperam numa fila - que se forma quando a taxa de atendimento é menor que a taxa de chegada de usuários -, são atendidos e saem do sistema após o serviço ser prestado.

De acordo com Hillier e Lieberman (2013), a formação de filas de espera é um fenômeno que ocorre quando a demanda se torna maior que a capacidade de fornecer um serviço em um determinado período.

Nesse contexto, surge o estudo da espera em todas as suas formas mais diversas (HILLIER; LIEBERMAN, 2013). A teoria das filas, como é conhecida, utiliza modelos de filas para representar diferentes casos e visa balancear os custos entre oferecer serviços e os custos de atrasos sofridos pelos usuários do sistema (ARENALES et al., 2007).

Moreira (2007) diz que a "Teoria das filas é um corpo de conhecimentos matemáticos, aplicado ao fenômeno das filas". Seu objetivo principal é desenvolver modelos matemáticos que permitam prever o comportamento de sistemas de prestações de serviços (MARINS, 2011). 
Para complementar Fogliatti\& Mattos (2007) afirmam que o estudo das filas é uma previsão do comportamento das mesmas com o propósito de regular a infraestrutura e o número de equipamentos, insumos e recursos tecnológicos necessários para evitar problemas por falta de recursos nesta fila de forma a manter os clientes satisfeitos.

\subsection{Caracterizações de um sistema de filas}

Para Mendonça (2014) um sistema com fila e composto por usuários, por canais ou postos de serviço/atendimento e por um espaço designado para a espera. Os usuários chegam em um determinado tempo, caracterizando o processo de chegada, para serem atendidos em determinado canal, posto de atendimento ou sequência de processo de manufatura, enquanto os postos estão ocupados os usuários aguardam em uma fila com espaço determinado para mesma.

As equações são classificadas baseadas nas seguintes características dos processos de chegada e de atendimentos aos clientes: As chegadas se processam segundo uma distribuição de Poisson com média $\lambda$ chegadas/tempo. Os tempos de atendimento seguem a distribuição exponencial de Poisson com média $\mu$. $\mathrm{O}$ atendimento à fila é feito por ordem de chegada. $\mathrm{O}$ numero de clientes potenciais é suficientemente grande para que a população possa ser considerada infinita, (ARENALES, 2007).

\subsection{Software ARENA}

O Arena é composto por um conjunto de módulos que atuam como comandos de uma linguagem de programação empregados para descrever uma aplicação real. Os elementos básicos para modelagem nesse software são os itens que representam as pessoas, objetos, processos, entre outras etapas que constituem as estações de trabalho (SILVA; PINTO; SUBRAMANIAN, 2007).

Segundo Pinho et al. (2009), o software Arena é um meio gráfico integrado de simulação. Nele não é preciso digitar nenhuma linha de código, visto que todo o procedimento de criação do modelo de simulação é gráfico e visual, e de modo integrado. A 6 ferramenta de simulação Arena possui todos os recursos para modelagem de processos, desenho e animação, análise estatística e análise de resultados.

De acordo com Pessanhaet al. (2011), para a construção de uma simulação é feito um modelo lógico-matemático que demonstra a dinâmica do sistema em estudo. Este modelo geralmente possui valores para tempos, distâncias e recursos disponíveis. 


\section{Metodologia}

A metodologia utilizada para o presente trabalho se deu através de uma abordagem quantitativa, de acordo com Creswell (2010), é caracterizada pela elaboração de questões e hipóteses em relação a determinado tema, sendo que as questões verificam as relações entre as variáveis formuladas e coletadas a partir de uma amostra, e as hipóteses, por sua vez, são as suposições que foram propostas e os possíveis relacionamentos entre as variáveis identificadas.

Os dados foram coletados por meio de uma pesquisa de campo. O estudo foi realizado em um supermercado na região do Cariri Paraibano, mais precisamente na cidade de Sumé, em um período entre 30 de outubro e 16 de novembro de 2019, resultando em um total de 5 avaliações, e teve como objetivo a análise de chegada de clientes em filas direcionadas aos caixas do estabelecimento citado anteriormente.

O processo de coleta de dados ocorreu entre 13:00:00 e 17:30:00 horas. A análise foi feita nos três caixas do estabelecimento, a fim de dar mais consistência a pesquisa. Para tal análise, foi feito o uso de um cronômetro que marcava o instante em que o cliente chegava na fila, quanto tempo esse cliente permanecia na fila e quanto tempo o mesmo passava em atendimento. Os dados obtidos estão na Tabela 1.

A pesquisa resultou em uma amostra de 164 pessoas, os dados foram tabulados no Microsoft Excel e compilados no Arena, software apresentado na disciplina em questão. Foi feito um estudo das filas para determinar alguns parâmetros, tais como:

- A taxa de utilização que é a relação entre o ritmo médio de chegada e o ritmo médio de atendimento, de acordo com Prado (2009). No caso de uma fila e vários atendentes:

$$
\rho=\frac{\lambda}{c \mu}
$$

Onde:

$\lambda$ : Ritmo de chegada de clientes em um fila

$\mu$ : Ritmo de atendimento de clientes

c: capacidade de atendimento ou quantidade de atendentes

- O intervalo médio de chegada, por definição, segundo Prado (2009):

$$
I C=\frac{1}{\lambda}
$$

Onde:

$\lambda$ : Ritmo de chegada de clientes em um fila 
- O tempo de atendimento, por definição, segundo Prado (2009):

$$
T A=\frac{1}{\mu}
$$

Onde:

$\mu$ : Ritmo de atendimento de clientes

\section{Resultados e discussões}

\subsection{Descrição do problema}

Nesta seção estão descritos todos os resultados inerentes à pesquisa, constando os dados coletados, as informações e inferências sobre o atual panorama da empresa. O problema em questão trata-se de uma análise de um possível desbalanceamento da estrutura de filas dos caixas de atendimento da empresa estudada, esse desequilíbrio pode causar perdas referentes ao tempo gasto pelos clientes quando os caixas não são o suficiente para o atendimento, bem como custos relacionados ao superdimensionamento dos caixas, gerando ociosidade dos mesmos e consequentemente desperdício.

\subsection{Dados de entrada}

Na tabela dos dados coletados, no dia 30/10/2019 totalizaram 25 clientes, no dia 09/11/2019 50 clientes, no dia 11/11/2019 43 clientes, no dia 14/11/2019 15 clientes e no dia 16/11/2019 31 clientes atendidos, gerando um total de 164 atendimentos, onde foi cronometrada a hora de chegada do cliente, tempo em que o cliente esperou em fila e a duração do atendimento no caixa, mediante esses dados foi gerado o tempo de atendimento médio, intervalo de chegada, o ritmo médio de atendimento de cada cliente, e o ritmo médio de chegada.

Tabela 1- Dados coletados (em minutos)

\begin{tabular}{|c|c|c|c|c|c|}
\hline Cliente & $\begin{array}{l}\text { Instante em que } \\
\text { o cliente chegou } \\
\text { (h:min:seg) }\end{array}$ & $\begin{array}{c}\text { Tempo que o } \\
\text { cliente passou } \\
\text { na fila } \\
\text { (h:min:seg) } \\
\end{array}$ & $\begin{array}{c}\text { Tempo de } \\
\text { duração do } \\
\text { atendimento } \\
\text { (h:min:seg) } \\
\end{array}$ & $\begin{array}{c}\text { Intervalo de } \\
\text { chegada }\end{array}$ & \\
\hline \multicolumn{5}{|c|}{ Data: 30/10/2019 } & \multirow{5}{*}{$\mathbf{A}$} \\
\hline 1 & $16: 29: 00$ & 00:00:10 & 00:00:42 & 00:04:00 & \\
\hline 2 & $16: 34: 00$ & 00:00:00 & $00: 01: 35$ & 00:05:00 & \\
\hline 3 & $16: 37: 00$ & 00:00:00 & 00:02:10 & 00:03:00 & \\
\hline 4 & 16:38:00 & 00:02:04 & $00: 00: 33$ & 00:01:00 & \\
\hline
\end{tabular}




\begin{tabular}{|c|c|c|c|c|}
\hline 5 & $16: 41: 00$ & 00:00:00 & 00:00:39 & 00:03:00 \\
\hline 6 & $16: 45: 00$ & 00:00:00 & $00: 00: 24$ & 00:04:00 \\
\hline 7 & $16: 46: 00$ & 00:00:14 & $00: 00: 26$ & 00:01:00 \\
\hline 8 & 16:48:00 & 00:01:33 & 00:01:57 & 00:02:00 \\
\hline 9 & 16:51:00 & $00: 00: 24$ & 00:01:06 & 00:03:00 \\
\hline 10 & 16:51:00 & $00: 01: 36$ & $00: 00: 17$ & 00:00:00 \\
\hline 11 & 16:53:00 & 00:00:59 & $00: 00: 32$ & 00:02:00 \\
\hline 12 & 16:55:00 & 00:00:00 & $00: 00: 39$ & 00:02:00 \\
\hline 13 & 16:56:00 & 00:00:00 & 00:00:29 & 00:01:00 \\
\hline 14 & $16: 56: 00$ & 00:01:28 & $00: 00: 23$ & 00:00:00 \\
\hline 15 & $16: 57: 00$ & 00:01:19 & $00: 00: 52$ & 00:01:00 \\
\hline 16 & 16:59:00 & 00:00:00 & 00:00:05 & 00:02:00 \\
\hline 17 & 17:00:00 & 00:00:00 & $00: 01: 54$ & 00:01:00 \\
\hline 18 & 17:01:00 & $00: 01: 26$ & $00: 00: 34$ & 00:01:00 \\
\hline 19 & 17:02:00 & 00:01:10 & $00: 00: 23$ & 00:01:00 \\
\hline 20 & 17:03:00 & 00:01:46 & 00:01:09 & 00:01:00 \\
\hline 21 & 17:09:00 & 00:00:53 & 00:00:52 & 00:06:00 \\
\hline 22 & 17:10:00 & 00:01:37 & $00: 05: 43$ & 00:01:00 \\
\hline 23 & $17: 17: 00$ & 00:00:19 & $00: 01: 25$ & $00: 07: 00$ \\
\hline 24 & 17:18:00 & 00:01:12 & 00:01:52 & 00:01:00 \\
\hline 25 & $17: 29: 00$ & 00:00:00 & $00: 00: 32$ & $00: 11: 00$ \\
\hline & Média $($ TA $)=$ & 0:01:05 & Média $($ IC $)=$ & 0:02:34 \\
\hline & $\begin{array}{l}\mu(\text { Ritmo médio } \\
\text { de } \\
\text { atendimento })=\end{array}$ & 0,92 & $\begin{array}{l}\lambda(\text { Ritmo médio } \\
\text { de chegada })=\end{array}$ & 0,39 \\
\hline
\end{tabular}

\section{Data: 09/11/2019}

\begin{tabular}{|r|r|r|r|r|}
\hline 26 & $13: 13: 00$ & $00: 00: 00$ & $00: 00: 31$ & $00: 03: 00$ \\
\hline 27 & $13: 17: 00$ & $00: 00: 00$ & $00: 00: 58$ & $00: 04: 00$ \\
\hline 28 & $13: 20: 00$ & $00: 00: 00$ & $00: 00: 16$ & $00: 03: 00$ \\
\hline 29 & $13: 21: 00$ & $00: 00: 00$ & $00: 01: 01$ & $00: 01: 00$ \\
\hline 30 & $13: 21: 00$ & $00: 00: 00$ & $00: 00: 43$ & $00: 00: 00$ \\
\hline 31 & $13: 23: 00$ & $00: 00: 00$ & $00: 00: 13$ & $00: 02: 00$ \\
\hline 32 & $13: 24: 00$ & $00: 00: 00$ & $00: 01: 15$ & $00: 01: 00$ \\
\hline 33 & $13: 24: 00$ & $00: 00: 00$ & $00: 00: 39$ & $00: 00: 00$ \\
\hline 34 & $13: 29: 00$ & $00: 00: 00$ & $00: 02: 21$ & $00: 05: 00$ \\
\hline 35 & $13: 30: 00$ & $00: 02: 35$ & $00: 00: 43$ & $00: 01: 00$ \\
\hline 36 & $13: 31: 00$ & $00: 00: 00$ & $00: 02: 10$ & $00: 01: 00$ \\
\hline 37 & $13: 34: 00$ & $00: 00: 00$ & $00: 01: 24$ & $00: 03: 00$ \\
\hline 38 & $13: 36: 00$ & $00: 00: 00$ & $00: 01: 15$ & $00: 02: 00$ \\
\hline 39 & $13: 37: 00$ & $00: 00: 00$ & $00: 01: 43$ & $00: 01: 00$ \\
\hline 40 & $13: 43: 00$ & $00: 00: 00$ & $00: 00: 27$ & $00: 06: 00$ \\
\hline 41 & $13: 44: 00$ & $00: 00: 00$ & $00: 00: 52$ & $00: 01: 00$ \\
\hline 42 & $13: 45: 00$ & $00: 00: 00$ & $00: 00: 31$ & $00: 01: 00$ \\
\hline 43 & $13: 46: 00$ & $00: 00: 00$ & $00: 00: 30$ & $00: 01: 00$ \\
\hline 44 & $13: 48: 00$ & $00: 00: 00$ & $00: 01: 40$ & $00: 02: 00$ \\
\hline
\end{tabular}




\begin{tabular}{|c|c|c|c|c|c|}
\hline 45 & $13: 49: 00$ & 00:00:00 & $00: 00: 35$ & 00:01:00 & \\
\hline 46 & 13:49:00 & 00:00:28 & $00: 00: 36$ & 00:00:00 & \\
\hline 47 & 13:50:00 & 00:00:00 & 00:01:06 & 00:01:00 & \\
\hline 48 & 13:50:00 & 00:00:00 & $00: 01: 54$ & 00:00:00 & \\
\hline 49 & 13:51:00 & $00: 02: 30$ & $00: 01: 22$ & 00:01:00 & \\
\hline 50 & 13:51:00 & 00:00:00 & $00: 00: 23$ & 00:00:00 & \\
\hline 51 & 13:53:00 & 00:00:00 & $00: 00: 46$ & 00:02:00 & \\
\hline 52 & 13:56:00 & 00:00:00 & 00:01:40 & 00:03:00 & \\
\hline 53 & 13:56:00 & 00:00:00 & $00: 01: 22$ & 00:00:00 & \\
\hline 54 & $13: 56: 00$ & $00: 01: 25$ & 00:01:09 & 00:00:00 & \\
\hline 55 & 13:57:00 & $00: 01: 11$ & $00: 00: 46$ & 00:01:00 & \\
\hline 56 & 13:57:00 & 00:00:00 & $00: 01: 50$ & 00:00:00 & \\
\hline 57 & 14:00:00 & 00:00:00 & $00: 00: 56$ & 00:03:00 & \\
\hline 58 & 14:02:00 & 00:00:00 & $00: 01: 34$ & 00:02:00 & \\
\hline 59 & 14:04:00 & 00:00:00 & $00: 01: 28$ & 00:02:00 & \\
\hline 60 & 14:06:00 & 00:00:00 & $00: 00: 25$ & 00:02:00 & \\
\hline 61 & 14:06:00 & $00: 00: 27$ & $00: 00: 49$ & 00:00:00 & \\
\hline 62 & 14:08:00 & 00:00:00 & $00: 01: 18$ & 00:02:00 & \\
\hline 63 & 14:09:00 & 00:00:00 & $00: 00: 58$ & 00:01:00 & \\
\hline 64 & 14:10:00 & 00:00:00 & 00:01:08 & 00:01:00 & \\
\hline 65 & 14:11:00 & 00:00:38 & 00:02:08 & 00:01:00 & \\
\hline 66 & 14:11:00 & $00: 02: 10$ & $00: 01: 47$ & 00:00:00 & \\
\hline 67 & 14:13:00 & $00: 01: 54$ & $00: 02: 30$ & 00:02:00 & \\
\hline 68 & 14:16:00 & 00:00:00 & $00: 00: 55$ & 00:03:00 & \\
\hline 69 & $14: 17: 00$ & 00:00:00 & $00: 00: 34$ & 00:01:00 & \\
\hline 70 & 14:20:00 & 00:00:00 & $00: 00: 56$ & 00:03:00 & \\
\hline 71 & $14: 20: 00$ & $00: 00: 15$ & $00: 00: 47$ & 00:00:00 & \\
\hline 72 & $14: 21: 00$ & 00:00:00 & $00: 01: 34$ & 00:01:00 & \\
\hline 73 & $14: 26: 00$ & 00:00:00 & $00: 01: 23$ & 00:05:00 & \\
\hline 74 & $14: 27: 00$ & 00:00:00 & $00: 00: 56$ & 00:01:00 & \\
\hline 75 & $14: 28: 00$ & 00:00:00 & 00:00:45 & 00:01:00 & \\
\hline & Média $($ TA $)=$ & 00:01:07 & Média $(\mathrm{IC})=$ & 00:01:34 & \\
\hline & $\begin{array}{l}\mu \text { (Ritmo médio } \\
\text { de atendimento) }\end{array}$ & 0,90 & $\begin{array}{l}\lambda(\text { Ritmo médio } \\
\text { de chegada })=\end{array}$ & 0,64 & \\
\hline \multicolumn{6}{|c|}{ Data: $11 / 11 / 2019$} \\
\hline 76 & 15:07:00 & 00:00:30 & $\begin{array}{r}00: 00: 31 \\
\end{array}$ & 00:07:00 & \multirow{10}{*}{$\mathrm{C}$} \\
\hline 77 & 15:09:00 & 00:00:58 & $00: 00: 23$ & 00:02:00 & \\
\hline 78 & $15: 13: 00$ & 00:00:00 & 00:00:29 & 00:04:00 & \\
\hline 79 & $15: 14: 00$ & 00:00:48 & 00:00:15 & 00:01:00 & \\
\hline 80 & $15: 15: 00$ & 00:00:18 & $00: 00: 54$ & 00:01:00 & \\
\hline 81 & 15:19:00 & 00:00:06 & 00:00:22 & 00:04:00 & \\
\hline 82 & $15: 20: 00$ & 00:00:07 & 00:00:58 & 00:01:00 & \\
\hline 83 & $15: 20: 00$ & 00:01:09 & 00:00:49 & 00:00:00 & \\
\hline 84 & $15: 27: 00$ & 00:00:20 & 00:01:08 & 00:07:00 & \\
\hline 85 & 15:29:00 & 00:00:15 & 00:00:49 & 00:02:00 & \\
\hline
\end{tabular}




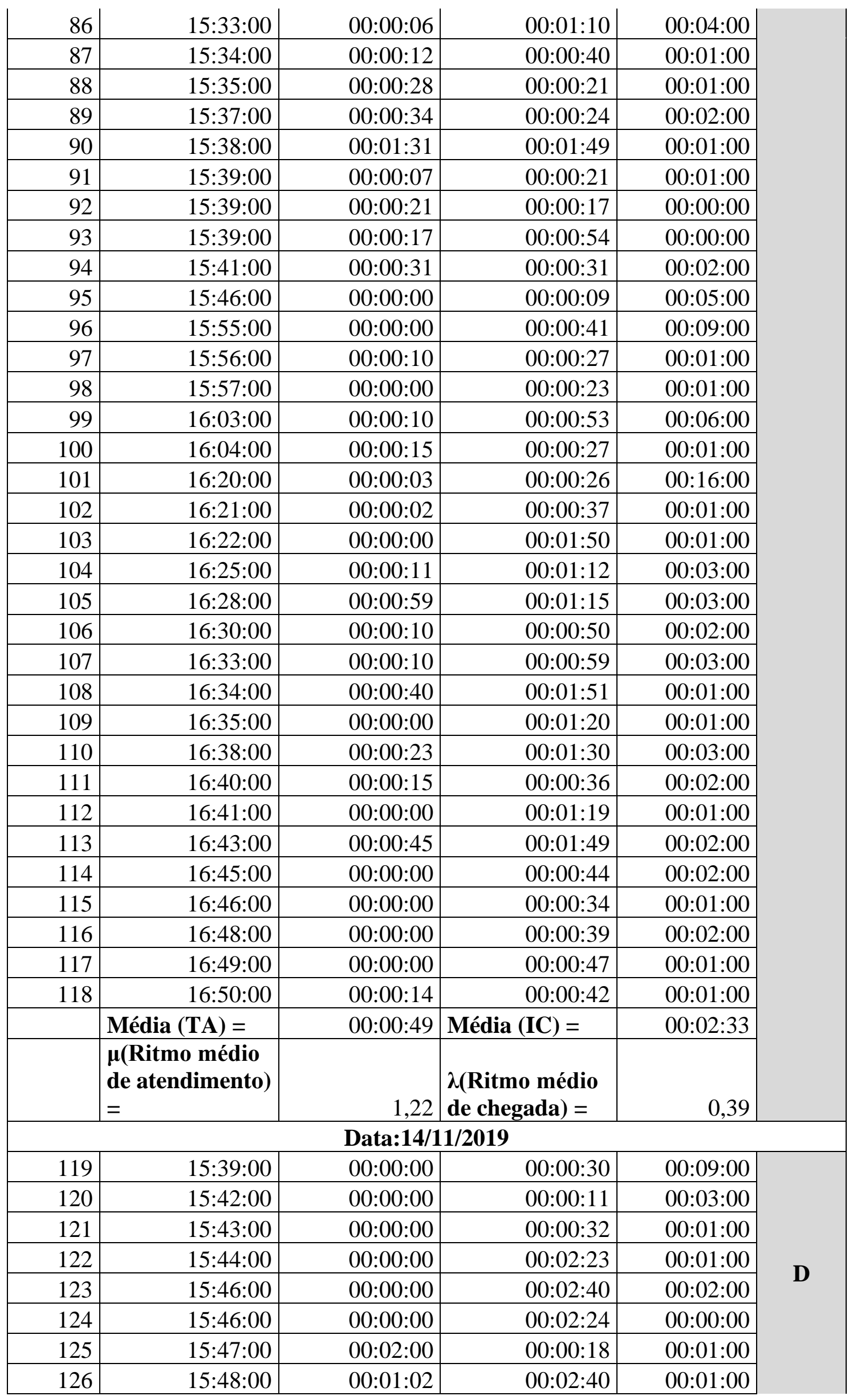




\begin{tabular}{|c|c|c|c|c|c|}
\hline 127 & $15: 50: 00$ & 00:01:00 & 00:01:22 & 00:02:00 & \\
\hline 128 & $15: 52: 00$ & 00:02:00 & 00:05:08 & 00:02:00 & \\
\hline 129 & 15:59:00 & 00:00:00 & 00:04:03 & 00:07:00 & \\
\hline 130 & 16:03:00 & 00:00:00 & 00:00:40 & 00:04:00 & \\
\hline 131 & 16:04:00 & 00:00:00 & 00:01:27 & 00:01:00 & \\
\hline 132 & $16: 05: 00$ & 00:01:01 & 00:00:40 & 00:01:00 & \\
\hline 133 & 16:06:00 & 00:01:10 & 00:00:23 & 00:01:00 & \\
\hline & Média $($ TA $)=$ & 00:01:41 & Média $(I C)=$ & 00:02:24 & \\
\hline & $\begin{array}{l}\mu(\text { Ritmo médio } \\
\text { de } \\
\text { atendimento })=\end{array}$ & 0,59 & $\begin{array}{l}\lambda(\text { Ritmo médio } \\
\text { de chegada) }=\end{array}$ & 0,42 & \\
\hline \multicolumn{6}{|c|}{ Data:16/11/2019 } \\
\hline 134 & $15: 05: 00$ & 00:00:00 & \begin{tabular}{|r|}
$00: 00: 35$ \\
\end{tabular} & 00:05:00 & \multirow{32}{*}{$\mathbf{E}$} \\
\hline 135 & 15:07:00 & 00:00:00 & 00:01:14 & 00:02:00 & \\
\hline 136 & 15:13:00 & 00:00:00 & $00: 01: 15$ & 00:06:00 & \\
\hline 137 & $15: 16: 00$ & 00:00:45 & 00:00:10 & 00:03:00 & \\
\hline 138 & 15:18:00 & 00:00:00 & 00:01:08 & 00:02:00 & \\
\hline 139 & 15:19:00 & $00: 00: 16$ & $00: 00: 13$ & 00:01:00 & \\
\hline 140 & $15: 21: 00$ & 00:00:00 & $00: 00: 26$ & 00:02:00 & \\
\hline 141 & $15: 23: 00$ & 00:00:00 & 00:01:01 & 00:02:00 & \\
\hline 142 & $15: 25: 00$ & 00:01:00 & 00:01:21 & 00:02:00 & \\
\hline 143 & $15: 27: 00$ & 00:00:32 & $00: 01: 22$ & 00:02:00 & \\
\hline 144 & $15: 28: 00$ & 00:00:30 & 00:01:16 & 00:01:00 & \\
\hline 145 & $15: 28: 00$ & 00:00:00 & $00: 00: 32$ & 00:00:00 & \\
\hline 146 & $15: 29: 00$ & 00:00:00 & 00:00:21 & 00:01:00 & \\
\hline 147 & $15: 29: 00$ & 00:00:00 & $00: 00: 31$ & 00:00:00 & \\
\hline 148 & $15: 29: 00$ & $00: 00: 25$ & $00: 04: 25$ & 00:00:00 & \\
\hline 149 & $15: 31: 00$ & 00:00:00 & $00: 01: 32$ & 00:02:00 & \\
\hline 150 & $15: 35: 00$ & 00:00:00 & 00:01:05 & 00:04:00 & \\
\hline 151 & 15:38:00 & 00:00:21 & $00: 00: 25$ & 00:03:00 & \\
\hline 152 & $15: 38: 00$ & 00:01:05 & $00: 00: 45$ & 00:00:00 & \\
\hline 153 & $15: 39: 00$ & 00:00:20 & $00: 01: 33$ & 00:01:00 & \\
\hline 154 & $15: 40: 00$ & 00:00:45 & 00:00:15 & 00:01:00 & \\
\hline 155 & $15: 43: 00$ & 00:00:00 & $00: 00: 34$ & 00:03:00 & \\
\hline 156 & $15: 44: 00$ & 00:00:00 & 00:00:26 & 00:01:00 & \\
\hline 157 & $15: 45: 00$ & 00:00:00 & 00:00:08 & 00:01:00 & \\
\hline 158 & $15: 46: 00$ & 00:00:00 & 00:00:31 & 00:01:00 & \\
\hline 159 & $15: 47: 00$ & $00: 00: 15$ & 00:00:30 & 00:01:00 & \\
\hline 160 & $15: 48: 00$ & 00:00:00 & 00:01:24 & 00:01:00 & \\
\hline 161 & $15: 49: 00$ & 00:00:00 & 00:00:33 & 00:01:00 & \\
\hline 162 & $15: 51: 00$ & 00:00:00 & 00:00:56 & 00:02:00 & \\
\hline 163 & $15: 55: 00$ & 00:00:00 & $00: 00: 58$ & 00:04:00 & \\
\hline \multirow[t]{2}{*}{164} & $15: 59: 00$ & 00:00:00 & 00:01:11 & 00:04:00 & \\
\hline & Média $($ TA $)=$ & 00:00:55 & Média $($ IC) $=$ & 00:01:54 & \\
\hline
\end{tabular}




\begin{tabular}{|l|l|l|r|r|} 
& $\begin{array}{l}\boldsymbol{\mu}(\text { Ritmo médio } \\
\text { de } \\
\text { atendimento })=\end{array}$ & 1,09 & $\begin{array}{l}\lambda(\text { Ritmo médio } \\
\text { de chegada })=\end{array}$ & 0,53
\end{tabular} \mid

Fonte: Autoria própria (2019)

\subsection{Modelo do sistema (Aplicação do Arena)}

Utilizando-se dos dados e separando-os entre os dias de coleta e os caixas analisados, foi utilizado o software ARENA a fim de simular os possíveis comportamentos dos sistemas propostos em dias de altas demandas e baixas demandas no período de tempo em que foram realizadas as cronometragens, verificando assim a fluidez e as margens que evitam a sobrecarga ou tempo de ociosidade dos caixas. A seguir estão dispostas as distribuições de probabilidade para os respectivos parâmetros em relação à chegada e atendimento desses clientes:

Figura 2- Distribuição da probabilidade nos dias de alta demanda (chegada de clientes)

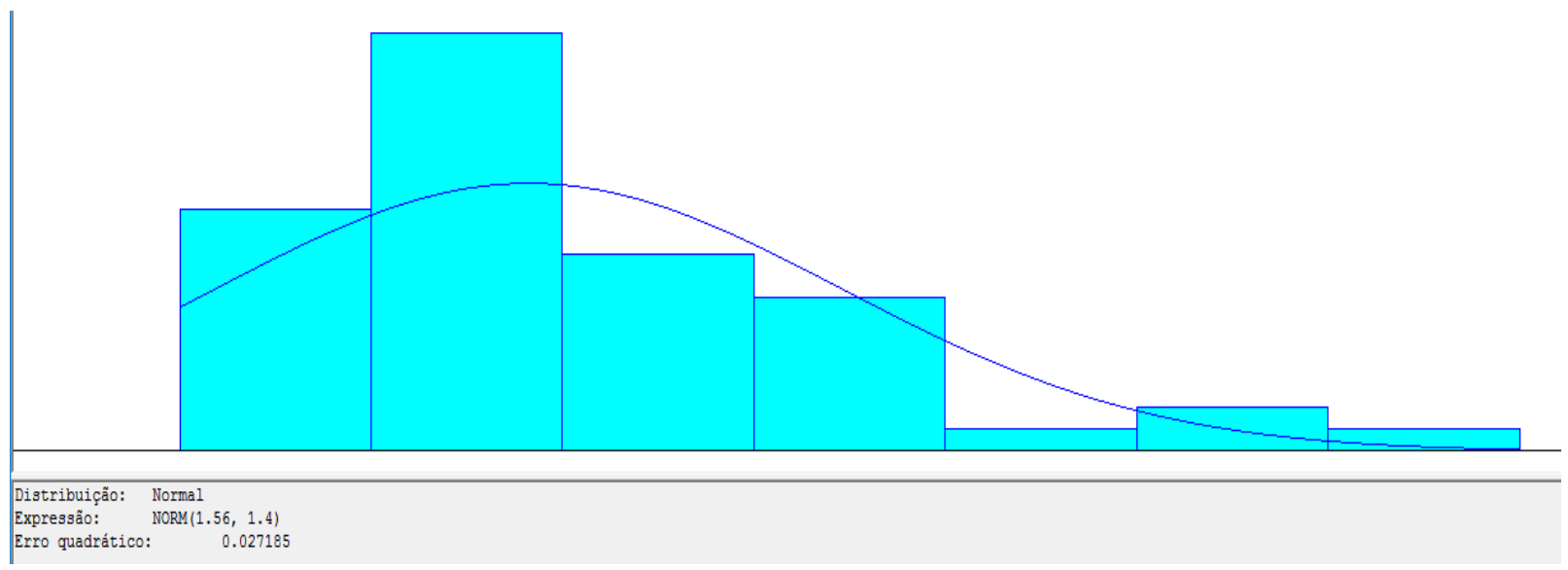

Fonte: Autoria própria (2019)

A figura a cima representa a distribuição normal da probabilidade de chega de clientes nos dias de altas de demandas.

Figura 3- Distribuição da probabilidade nos dias de alta demanda (atendimento) 


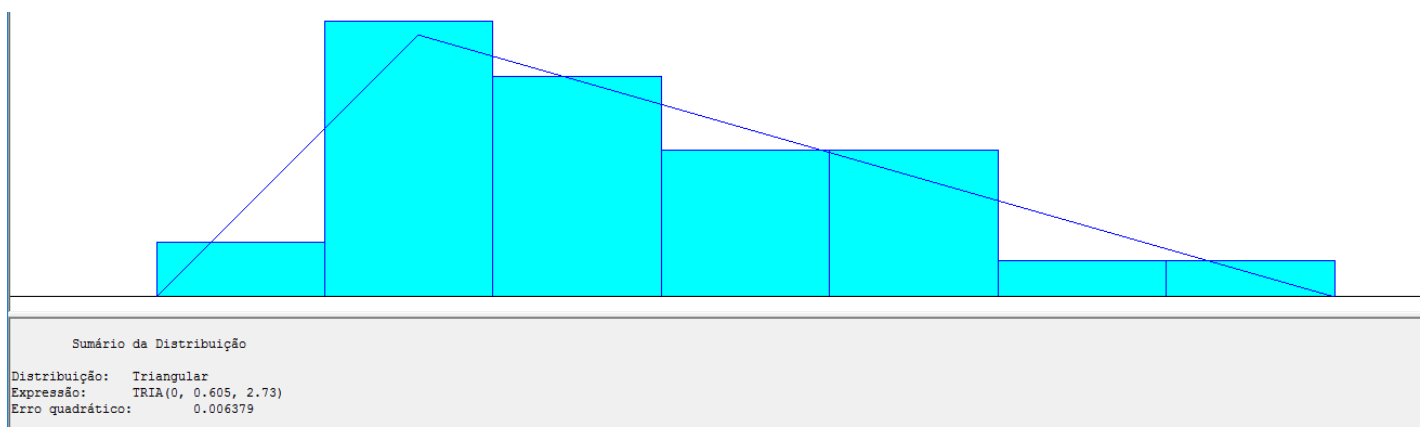

Fonte: Autoria própria (2019)

A figura a cima representa uma distribuição triangular da probabilidade de atendimento nos dias de altas demandas.

Figura 4- Distribuição da probabilidade nos dias de baixa demanda (chegada de clientes)

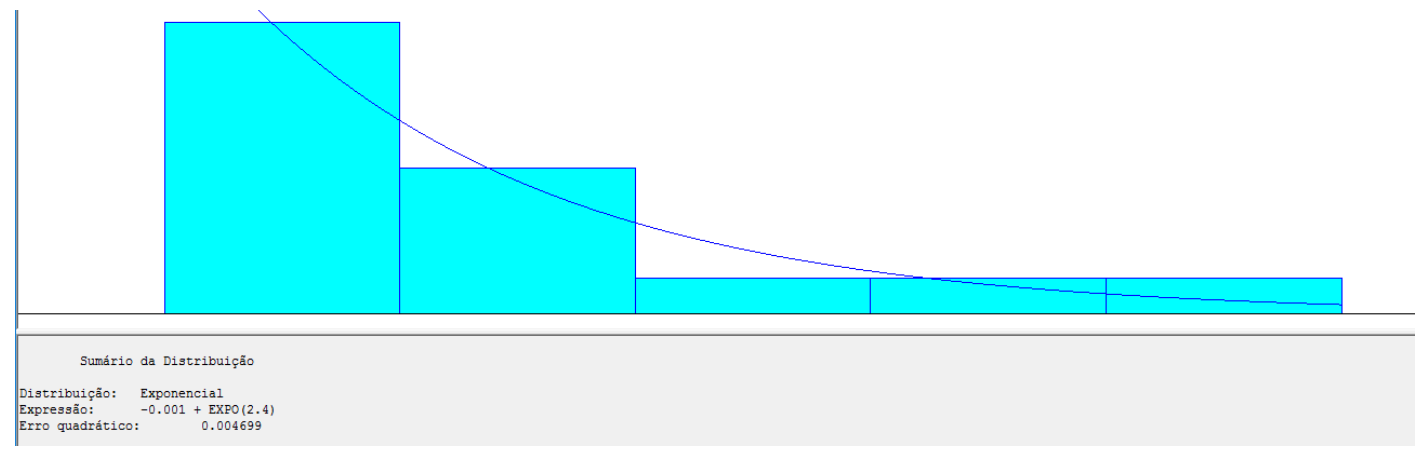

Fonte: Autoria própria (2019)

A figura acima representa uma distribuição exponencial para a chegada de clientes em dias de baixa demanda.

Figura 5- Distribuição da probabilidade nos dias de alta demanda (atendimento)

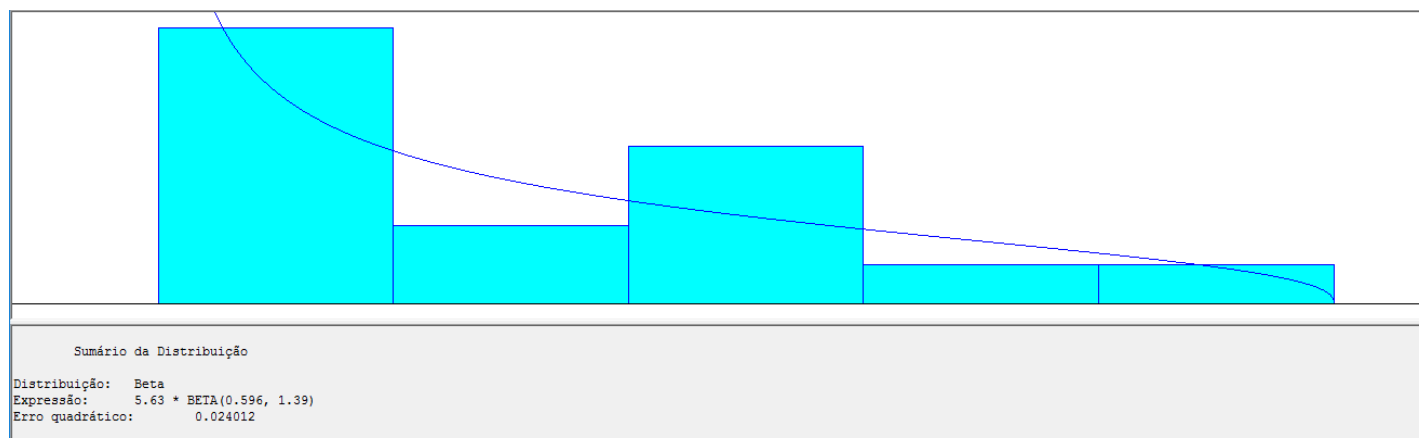

Fonte: Autoria própria (2019)

A figura a cima representa um histograma de distribuição BETA para a probabilidade de atendimento de clientes em dias de baixa demanda 
Após encontrar a distribuição de probabilidade, encontrou-se a lógica do modelo estudado.

Figura 6- Lógica do modelo para 3 caixas em dias de alta demanda.

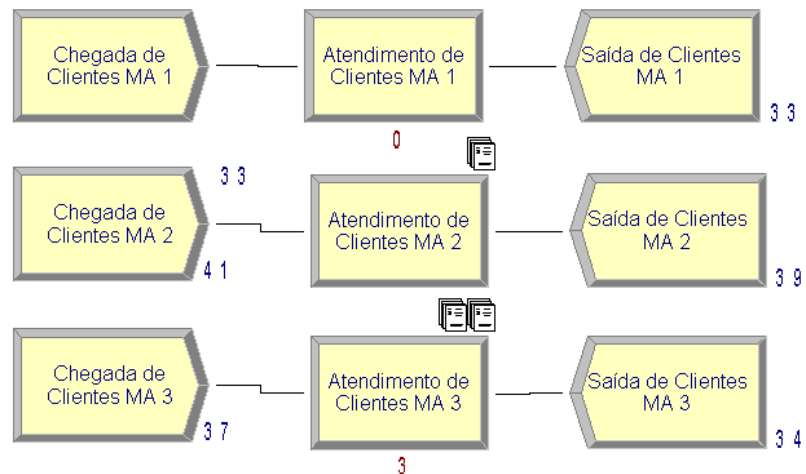

Fonte: Autoria própria (2019)

Figura 7- Lógica do modelo para 2 caixas em dias de alta demanda.

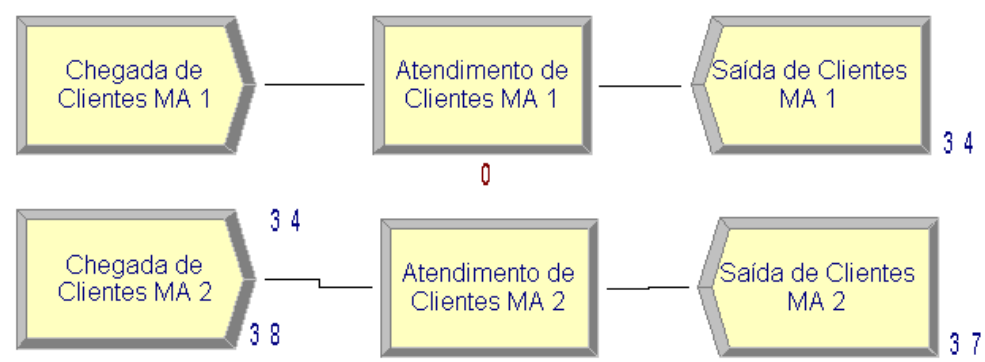

Fonte: Autoria própria (2019)

Figura 8- Lógica do modelo para 3 caixas em dias de baixa demanda.

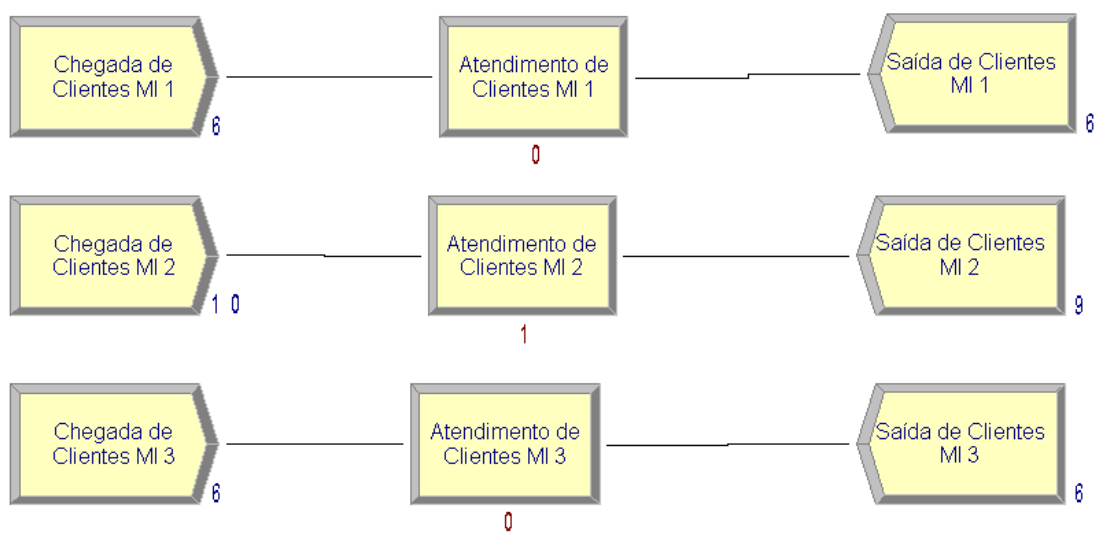

Fonte: Autoria própria (2019)

Figura 9- Lógica do modelo para 2 caixas em dias de baixa demanda. 


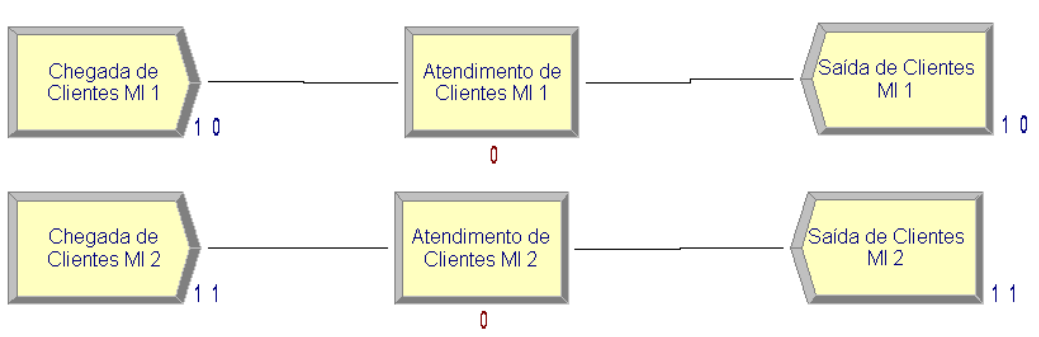

Fonte: Autoria própria (2019)

Figura 10: Lógica do modelo para 1 caixa em dias de baixa demanda.

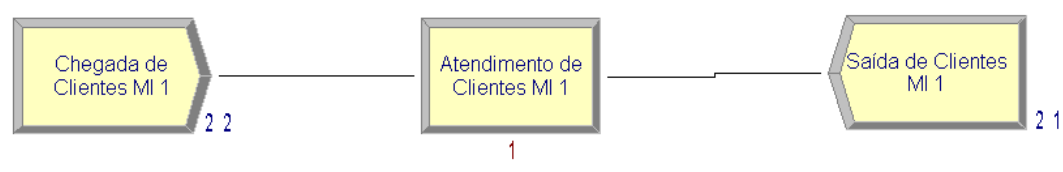

Fonte: Autoria própria (2019)

\subsection{Análise dos resultados}

Tabela 2- Simulação com 3 caixas nos dias de baixa demanda (em minutos)

\begin{tabular}{|c|c|c|c|}
\hline & Caixa 1 & Caixa 2 & Caixa 3 \\
\hline $\begin{array}{c}\text { Tempo de } \\
\text { espera }\end{array}$ & 0.00 & 0.18 & 0.00 \\
\hline $\begin{array}{c}\text { Tempo de } \\
\text { atendimento }\end{array}$ & 2.17 & 1.85 & 1.54 \\
\hline $\begin{array}{c}\text { Tempo do } \\
\text { sistema }\end{array}$ & 2.17 & 2.03 & 1.54 \\
\hline $\begin{array}{c}\text { Número } \\
\text { médio de } \\
\text { clientes no } \\
\text { sistema }\end{array}$ & 0.22 & 0.33 & 0.15 \\
\hline $\begin{array}{c}\text { Taxa de } \\
\text { utilização }\end{array}$ & Atendente 1 & Atendente 2 & Atendente 3 \\
\cline { 2 - 4 } & 0.22 & 0.30 & 0.15 \\
\hline
\end{tabular}

Fonte: Autoria própria (2019)

A tabela acima representa a situação atual de forma simulada em dias de baixa demanda, onde podemos notar um tempo de espera baixo em todos os caixas e um tempo de ociosidade bastante elevado.

Tabela 3- Simulação com 3 caixas nos dias de alta demanda (em minutos)

\begin{tabular}{|c|c|c|c|}
\hline & Caixa 1 & Caixa 2 & Caixa 3 \\
\hline $\begin{array}{c}\text { Tempo de } \\
\text { espera }\end{array}$ & 0.01 & 0.01 & 0.01 \\
\hline
\end{tabular}




\begin{tabular}{|c|c|c|c|}
$\begin{array}{c}\text { Tempo de } \\
\text { atendimento }\end{array}$ & 0.02 & 0.01 & 0.02 \\
\hline $\begin{array}{c}\text { Tempo do } \\
\text { sistema }\end{array}$ & 0.02 & 0.02 & 0.03 \\
\hline $\begin{array}{c}\text { Número médio } \\
\text { de clientes no } \\
\text { sistema }\end{array}$ & 0.98 & 1.15 & 1.22 \\
\hline $\begin{array}{c}\text { Taxa de } \\
\text { utilização }\end{array}$ & Atendente 1 & Atendente 2 & Atendente 3 \\
\cline { 2 - 4 } & 0.68 & 0.73 & 0.76 \\
\hline
\end{tabular}

Fonte: Autoria própria (2019)

A tabela acima representa a simulação dos dados reais em dias de alta demanda, podendo-se notar a taxa de utilização em um nível intermediário próximo de $70 \%$ uso do atendente.

Tabela 4- Simulação com 2 caixas nos dias de baixa demanda (em m
\begin{tabular}{|c|c|c|}
\hline $\begin{array}{c}\text { Tempo de } \\
\text { espera }\end{array}$ & Caixa 1 & Caixa 2 \\
\hline $\begin{array}{c}\text { Tempo de } \\
\text { atendimento }\end{array}$ & 2.24 & 0.12 \\
\hline $\begin{array}{c}\text { Tempo do } \\
\text { sistema }\end{array}$ & 2.37 & 1.52 \\
\hline $\begin{array}{c}\text { Número médio } \\
\text { de clientes no } \\
\text { sistema }\end{array}$ & 0.39 & 0.32 \\
\hline Taxa de & Atendente 1 & Atendente 2 \\
\cline { 2 - 4 } utilização & 0.37 & 0.28 \\
\hline
\end{tabular}

Fonte: Autoria própria (2019)

A tabela acima representa a análise dos dados em dias de baixa demanda simulando a utilização de apenas dois caixas, onde podemos notar uma menor ociosidade em relação a situação real, três caixas.

Tabela 5- Simulação com 2 caixas nos dias de alta demanda (em minutos)

\begin{tabular}{|c|l|l|}
\hline & Caixa 1 & Caixa 2 \\
\hline $\begin{array}{c}\text { Tempo de } \\
\text { espera }\end{array}$ & 0.01 & 0.02 \\
\hline $\begin{array}{c}\text { Tempo de } \\
\text { atendimento }\end{array}$ & 0.02 & 0.02 \\
\hline
\end{tabular}




\begin{tabular}{|c|l|l|} 
Tempo do & \\
sistema & 0.03 & 0.04 \\
\hline $\begin{array}{c}\text { Número médio } \\
\text { de clientes no } \\
\text { sistema }\end{array}$ & 0.94 & 1.53 \\
\hline $\begin{array}{c}\text { Taxa de } \\
\text { utilização }\end{array}$ & Atendente 1 & Atendente 2 \\
\cline { 2 - 3 } & 0.67 & 0.81 \\
\hline
\end{tabular}

Fonte: Autoria própria (2019)

A tabela acima representa a simulação dos dados em dias de alta demanda, quando retirado um caixa de atendimento, podendo notar uma alta taxa de utilização, porém, com uma boa margem de segurança para casos extremos. A simulação com apenas um caixa se torna inviável pois os valores quando de utilização se tornam superiores ao suportado pelo sistema.

Tabela 6 - Simulação com 1 caixas nos dias de baixa demanda (em minutos)

\begin{tabular}{|c|l|}
\hline & Caixa 1 \\
\hline $\begin{array}{c}\text { Tempo de } \\
\text { espera }\end{array}$ & 1.90 \\
\hline $\begin{array}{c}\text { Tempo de } \\
\text { atendimento }\end{array}$ & 1.75 \\
\hline $\begin{array}{c}\text { Tempo do } \\
\text { sistema }\end{array}$ & 3.66 \\
\hline $\begin{array}{c}\text { Número } \\
\text { médio de } \\
\text { clientes no } \\
\text { sistema }\end{array}$ & 1.31 \\
\hline $\begin{array}{c}\text { Taxa de } \\
\text { utilização }\end{array}$ & Atendente 1 \\
\cline { 2 - 2 }
\end{tabular}

Fonte: Autoria própria (2019)

A tabela acima representa a análise de dados em dias de baixa demanda simulando a utilização de apenas um caixa, onde podemos notar uma menor ociosidade em relação aos modelos de dois e três caixas, e com isso aumentando consideravelmente os tempos de sistema, espera e atendimento.

\subsection{Propostas de Melhorias}


Após a análise dos dados e das simulações feitas no ARENA, as informações coletadas propuseram a retirada de um caixa de atendimento do sistema, permanecendo assim com apenas 2 caixas, devido aos elevados percentuais de ociosidade quando em dias de baixa, e ainda em dias de altas demandas, no período de tempo analisado, mantendo uma margem de segurança em relação a qualidade de atendimento, ou seja o sistema possuía um superdimensionamento, trabalhando assim bem abaixo da capacidade máxima. A propositura de 2 caixas trará uma maior utilização sem exceder os limites nos dias de altas demandas gerando menor custo para empresa e mantendo um limite de segurança em eventos de altas inesperadas, podendo ainda, aproveitar o funcionário que estaria ocioso em outras funções nesse período.

\section{Considerações finais}

A proposta para a iniciação deste trabalho foi usar a teoria das filas para simplificar, otimizar e avaliar os parâmetros de efetividade do sistema de uma fila que se forma nos acesso de três canais de atendimento (caixa) de um Supermercado localizado na região do Cariri no estado da Paraíba. Com o intuito de apresentar modelos que melhorassem a satisfação do cliente e reduzissem os custos. Através do entendimento de teoria das filas e com a ajuda de ferramentas como o Arena e o Excel foi possível extrair dados a respeito da situação atual do sistema para o espaço de tempo em que foram realizadas as cronometragens e perceber a ociosidade presente no mesmo.

Os resultados alcançados comprovam que o sistema em estudo está superdimensionado, a simulação realizada com três atendentes é considerada desvantajosa por trazerem custos operacionais para organização. Dessa forma, a situação proposta se torna viável, apesar de aumentar os níveis de utilização de caixas, os parâmetros continuam aceitáveis.

Esse estudo deve ser aprofundado, pois ele abrangeu um pequeno período de tempo do funcionamento do mercado, havendo possibilidade de resultados mais robustos se fosse realizado em horários e dias prolongados.

\section{REFERÊNCIAS}

ARENALES, M. et al. Pesquisa operacional. 1. ed., 6. reimpr. Rio de Janeiro: Elsevier, 2007.

CAIXETA-FILHO, J. V.. Pesquisa operacional: técnicas de otimização aplicadas a sistemas agroindustriais. São Paulo: Atlas, 2004.

FOGLiATTI, M. C.; MATTOS, N. M. C. Teoria de Filas. Editora Interciência. Rio de Janeiro, 2007. 
HILLIER, F. S.; LIEBERMAN, G. J. Introdução à pesquisa operacional. 9. ed. Porto Alegre: AMGH, 2013.

LOVELOCK, C.; WRIGHT, L. Serviços: marketing e gestão. São Paulo: Saraiva, 2002.

MOREIRA, D. A. Pesquisa Operacional: curso introdutório. São Paulo: Thomson Learning, 2007.

MENDONÇA, E. Teoria de filas markovianas e aplicações, 2014.

MARINS, F. A. S. Introdução à Pesquisa Operacional. São Paulo: Cultura Acadêmica, Pró Reitoria de Graduação, 2011.

CRESWELL, J. W. Projeto de pesquisa métodos qualitativo, quantitativo e misto. Artmed, 2010.

MORABITO, R.; LIMA, F. C. R. Um Modelo Para Analisar O Problema De Filas Em Caixas De Supermercados: Um Estudo De Caso. Pesqui. Oper. vol.20 no.1 Rio de Janeiro June 2000. Disponível em:<http://www.scielo.br/scielo.php?pid=S0101-74382000000100007\&script=sci_arttext\&tlng=pt $>$ Acesso em 26 nov. 2019.

PESSANHA, A. M. B.; FILHO S. M. D. R.; MELO, N. A. F. Estudo da aplicação do software arena em um contrato de prestação de serviço de manutenção de instrumentação. Rio de Janeiro, 2011.

PINHO, B.; CAPELLI, C.; LIMA, L.; NASCIMENTO, L.; SENNA, P.; PAIM, R. Metodologias e Ferramentas para Simulação de Processos. Rio de Janeiro: Universidade Federal do Rio de Janeiro, 2009.

PRADO, D. S.. Teoria das filas e da simulação. 4. Ed.. Nova lima: INDG Tecnologia e Serviços Ltda, Minas gerais, 2009.

SANTOS, J. B. D. et al. Estudo Da Teoria Das Filas Em Uma Unidade Farmacêutica Situada No Munícipio De Abaetetuba, Pará. 2016. Disponível em:. Acesso em: 26 nov. 2019.

SILVA, L. M. F., PINTO, M. G., SUBRAMANIAN, A. Utilizando o software Arena como ferramenta de apoio ao ensino em Engenharia de Produção. Foz do Iguaçu, 2007.

PESSANHA, A. M. B.; FILHO S. M. D. R.; MELO, N. A. F. Estudo da aplicação do software arena em um contrato de prestação de serviço de manutenção de instrumentação. Rio de Janeiro, 2011. 